\title{
Nomenclature for Kidney Function from KDIGO: Shortcomings of Terminology Oversimplification
}

\author{
Alberto Palazzuolia Kristen M. Tecson ${ }^{b, c}$ Claudio Ronco $^{d}$ \\ Peter A. McCullough ${ }^{c}$ \\ aDepartment of Internal Medicine, Cardiovascular Diseases Unit, Le Scotte Hospital University of Siena, Siena,

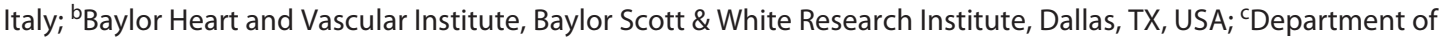 \\ Internal Medicine, Texas A\&M University Health Science Center College of Medicine, Dallas, TX, USA; ${ }^{\mathrm{d} D e p a r t m e n t}$ \\ of Nephrology, San Bortolo Hospital and International Renal Research Institute of Vicenza (IRRIV), Vicenza, Italy
}

\author{
Keywords \\ Kidney · Renal function - Cardiorenal syndrome · Biomarkers
}

\section{Abstract}

The recent Kidney Disease: Improving Global Outcomes (KDIGO) consensus conference proposed a universal nomenclature calling for "Kidney Disease" (KD) to be applied to every form of kidney dysfunction, regardless of etiology. We recognize that the estimated glomerular filtration rate and urine albumin:creatinine ratio are limited in their application to the broad spectrum of KD. However, there are additional in vitro and advanced diagnostic options that can help identify the underlying cause of KD and inform about prognosis and management. While the overarching benefit of generalizing $\mathrm{KD}$ as a medical problem lies with screening and detection, the downsides attributable to a nonexact diagnosis (i.e., unclear prognosis and management strategy) are considerable. Finally, the terms "acute kidney injury" and "worsening renal function" are currently used interchangeably by nephrologists and cardiologists alike, and a universal adoption of one term will likely be a sizeable challenge. To be of great-

karger@karger.com www.karger.com/crm

Karger $\stackrel{\text { ' }}{5}$
(C) 2021 The Author(s)

Published by S. Karger AG, Basel

This is an Open Access article licensed under the Creative Commons Attribution-NonCommercial-4.0 International License (CC BY-NC) (http://www.karger.com/Services/OpenAccessLicense), applicable to the online version of the article only. Usage and distribution for commercial purposes requires written permission. er benefit, we propose KD be used as a starting point and that the etiology and other epigenetic determinants of illness continue to be evaluated and characterized.

(c) 2021 The Author(s)

Published by S. Karger AG, Basel

\section{Introduction}

Kidney disease (KD) is an increasing and underestimated disorder occurring in a large portion of patients, disproportionately affecting those with cardiovascular (CV) comorbidities. Members of the Kidney Disease: Improving Global Outcomes (KDIGO) consensus conference recently suggested to adopt a common nomenclature surrounding any form of kidney dysfunction [1]. This new movement arose due to the mounting evidence that similar KD subtypes with the same or similar diagnostic and clinical significance are differentially termed and misunderstood [2]. Therefore, it is the hope that universal medical terminology in KD may improve health status knowledge for patients with these ailments. 


\section{Gaps in Terminology Simplification}

Although the effort to standardize nomenclature and classification should be commended, retaining some levels of differentiation may be most appropriate to discern the broad etiology and pathophysiological mechanisms leading to kidney dysfunction and progression. This is relevant for both acute and chronic $\mathrm{KD}$, with the trajectories depending on different $\mathrm{KD}$ subtypes, external features, and factors responsible for kidney impairment [3]. Indeed, CKD signifies various stimuli ranging from common CV risk factors including genetics to prior infective systemic or metabolic diseases and chronic hemodynamic alterations. Within each of these determinants, we can identify an enormous cluster of pathogenic features and potential treatment targets $[4,5]$. In clinical practice, relying only on creatinine and glomerular filtration rate (GFR), CKD becomes homogenized, and its diagnosis/ staging, prognosis, and management become inexact. Creatinine and GFR are both markers of late kidney damage, unable to recognize CKD in its earliest stage or identify the precise parenchymal tubular or glomerular damage [6]. Therefore, with the current approach, physicians are powerless to identify the causes of hemodynamic derangement. Thus, there are several questions that need to be addressed concerning which laboratory biomarkers to monitor, the exact prevalence in different $\mathrm{CV}$ and metabolic diseases in the real world, and the related clinical significance. The consensus document from the recent KDIGO conference endorses GFR and albuminuria as essential. This approach contains several pitfalls: (1) GFR and albuminuria may deteriorate on different trajectories according to course of treatment; (2) GFR reflects one dimension of kidney function deterioration caused by many triggers and does not distinguish the source of primary kidney damage; conversely, albuminuria is mainly a marker of increased glomerular permeability and failure of tubular reabsorption; (3) neither biomarkers identify the real kidney damage nor KD etiology (i.e., genetic, vascular, toxic, metabolic, septic, or parenchymal) $[7,8] ;$ (4) $\mathrm{CKD}$ and acute $\mathrm{KD}$ have different development, time courses (slow vs. sudden), and related systemic and CV complications; (5) finally, adoption of uniform nomenclature could create risk for erroneous disease classification and prognostication. Indeed, the application of broad terminology does not necessarily translate to improved patient awareness. In fact, patient outcomes are largely driven by patient compliance, which is generally improved when patients have a strong understanding of their exact condition.

\section{Time Course and Severity Assessment}

In their summary document, KDIGO purposed to delete the terms "renal" and "end stage renal disease" (ESRD) in favor of kidney and kidney failure, respectively. Conversely, we believe that renal is an appropriate term widely used in the European Union and North American countries, largely due to the influence of the Latin language. Perhaps the terms may be less employed in the Asian and African countries, where different cultures and spelling are common. The "renal" diction remains suitable for functional evaluation of $\mathrm{KD}$ during temporal laboratory assessment and in more advanced KD stages in which the term "failure" appears too coarse and does not adequately represent the KD severity [9]. Kidney failure may have different clinical manifestations: incapacity to produce adequate urine volume, systemic symptoms (weakness, dizziness, asthenia, and reduced exercise tolerance), poor quality of life, or even paucisymptomatic in those with long KD history and mild evolution. Conversely, "ESRD" defines an exact health state via laboratory and clinical thresholds (i.e., GFR $<15 \mathrm{~mL} / \mathrm{m}^{2}$ and renal replacement therapy). Notably, the restriction to GFR and albuminuria for kidney function evaluation ignores other laboratory measures capable to recognize kidney site damage. Of note, previous AKIN/RIFLE documents and scientific societies proposed a more integrated laboratory evaluation platform, which is particularly useful in the acute setting. Due to their diagnostic and prognostic relevance, some of these biomarkers (NAG, NGAL, KIM-1, and cystatin C) have been extended for use in the chronic setting to possibly correct the primary kidney damage and stall disease progression [10]. The other reason the broad application of "kidney" is advocated by the KDIGO conference attendees is its correspondence with histological and cellular derangements recruited in bioptic examination. Theoretically, this could summarize clinical and pathological features. Unfortunately, the findings from these assessments are not always in agreement in practice. For example, anatomic disarray has poor correlation with kidney function as measured by albuminuria and GFR. Additionally, severe microscopic alterations have only a modest relation with urinary production capacity. Further, inside biopsied tissues are different manifestations and wide histological panels revealing the evolution of kidney dysfunction and severity [11]. Finally, only a small percentage of patients with acute kidney injury (AKI)/CKD are referred for kidney biopsy; otherwise, most subjects with KD are monitored and treated with- 
out invasive examination. Due to these concerns, the extensive use of "KD" outside of nephrology may be misleading (Fig. 1).

\section{Conflict with Cardiorenal Syndrome Definition}

The complete removal of the term "renal" from this discipline will fail to adequately describe the concept of reciprocal renal and cardiac damage occurring in cardiorenal syndrome. It is universally recognized that any hemodynamic alterations due to $\mathrm{CV}$ disease could result in kidney damage and functional impairment $[12,13]$. Likewise, underlying $\mathrm{KD}$ can induce cardiac dysfunction as well as structural damage. This bidirectional relationship exists in both acute and chronic settings, even if morphological dysfunction occurs at different times. Thus, the substitution of the joint "cardiorenal" term with distinct "heart" and "kidney" does not appear to be beneficial in practice. Beyond kidney and circulatory dysfunction, the complex cardiorenal axis encompasses several mechanisms including nephron loss, tubular damage, salt and water retention, renal congestion, and glomerulosclerosis. Activation of the neuroendocrine system is another feature at play in both organs: in the kidneys, it affects sodium and water resorption, intrarenal blood flow redistribution, reduced medullary blood flow, glomerulosclerosis, tubular fibrosis, and efferent and afferent arterial vasoconstriction. In the heart, it increases cardiac workload and filling pressure, systemic vasoconstriction, and cardiac mass, inducing myocardial fibrosis, endothelial dysfunction, and atherosclerotic process. Consequently, patients with severe neurohormonal abnormalities are more likely to have systemic congestion, require a higher diuretic dose, and have higher CV risk burden [3]. In other words, the renal derangements of cardiorenal syndrome may be the result of systemic and local hemodynamic modification, intrarenal perfusion alteration, and parenchymal damage. Thus, KD in this setting may simply reflect sicker patients with advanced refractory HF and loss of intrarenal autoregulation. Accordingly, the clinical relevance of blood urea nitrogen (BUN) in this setting has been confirmed because it reflects renin angiotensin axis activity. Although it is not specifically a renal marker, urea metabolism depends on 3 renal variables: BUN concentration at the proximal tubule level, related to systemic urea production; urea velocity and reabsorption at the distal tubule; and urea transport and concentration in the inner medullary. Classifying the dominant pathophysiologic mechanism may require as-

Nomenclature for Kidney Function from KDIGO

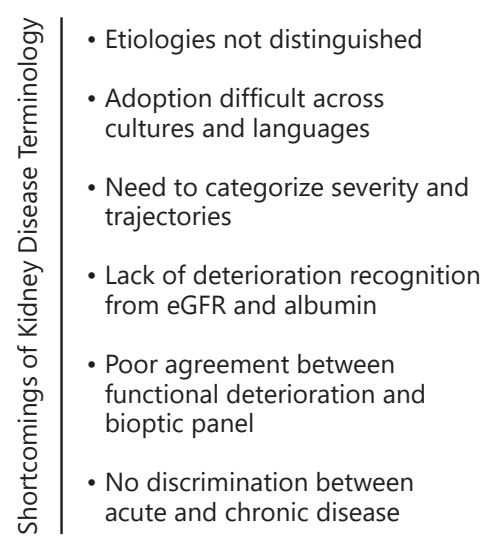

Fig. 1. Shortcomings of kidney dysfunction terminology: the same term is applied to different settings without distinguishing between the specific clinical scenarios or renal function trajectories. Thus, the suggested terminology may be expanded according to laboratory biomarker evaluation and dysfunction severity.

sessment of several biomarkers. For example, GFR fluctuates and creatinine is overexpressed and may be unpredictable in the setting of AKI. In this setting, there is no universal agreement as to which biomarkers should be used to monitor kidney function [14]. The terms AKI and worsening renal function are interchangeably used by nephrologists and cardiologists alike. In this sense, the AKIN criteria are the only guidelines providing a standardized description for the early diagnosis and severity of AKI. The introduction of a universal definition of AKI/ worsening renal function based on biomarkers to identify the primary site of damage (i.e., hemodynamic, glomerulus, tubule, or collecting duct) would be a challenge for standardization [15]. Thus, the term "renal" should not be orphaned in any lexicon.

\section{Homogenization of Terms}

Nomenclature homogenization may have potential advantages in screening and detection of KD. However, its oversimplification may lead to an underestimation of dysfunction severity. The scientific community's debate over the most appropriate definitions for CKD and AKI requires standardized laboratory measures to classify stages, severity, and kidney site damage. Going forward, perhaps the term "kidney" may be the most appropriate 
for patients with well-identified macroscopic and microscopic kidney alterations reflecting the precise disease stage. This evaluation may be applicable following the extensive use of imaging and minimally invasive tools to examine anatomic and pathologic panels. Conversely, the term "renal," to describe functional capacity, may be utilized when evaluating laboratory biomarkers, such as albuminuria and GFR, creatinine, or BUN. This distinction could lead to improved anatomical and functional classification, providing richer information for patients and clinicians.

\section{Conclusions}

The recent proposition from the KDIGO consensus conference arises from the need to achieve a common medical language and approach to care. However, the proposed nomenclature may be an oversimplification as there are clinical scenarios in which specific language could help define the broad etiology and pathophysiological processes. The extensive use of in vitro and other advanced diagnostics can be used to identify the underlying cause of KD. Accordingly, researchers should arrange a common definition for both chronic and acute $\mathrm{KD}$, using a specific set of laboratory measures to identify the location and severity of kidney damage.

\section{Conflict of Interest Statement}

There are no conflicts of interest.

\section{References}

1 Levey AS, Eckardt KU, Dorman NM, Christiansen SL, Cheung M, Jadoul M, et al. Nomenclature for kidney function and diseaseexecutive summary and glossary from a kidney disease: improving Global Outcomes (KDIGO) consensus conference. Eur Heart J. 2020 Dec 21;41(48):4592-8.

2 Sarnak MJ, Levey AS, Schoolwerth AC, Coresh J, Culleton B, Hamm LL, et al. Kidney disease as a risk factor for development of cardiovascular disease: a statement from the American Heart Association Councils on Kidney in Cardiovascular Disease, High Blood Pressure Research, Clinical Cardiology, and Epidemiology and Prevention. Circulation. 2003 Nov; 108(5):2154-69.

3 Mullens W, Damman K, Testani JM, Martens P, Mueller C, Lassus J, et al. Evaluation of kidney function throughout the heart failure trajectory: a position statement from the Heart Failure Association of the European Society of Cardiology. Eur J Heart Fail. 2020 Apr; 22(4):584-603.

4 McCullough PA, Li S, Jurkovitz CT, Stevens LA, Wang C, Collins AJ, et al. CKD and cardiovascular disease in screened high-risk volunteer and general populations: the Kidney Early Evaluation Program (KEEP) and $\mathrm{Na}-$ tional Health and Nutrition Examination Survey (NHANES) 1999-2004. Am J Kidney Dis. 2008 Apr;51(4 Suppl 2):S38-45.
5 Palazzuoli A, Lombardi C, Ruocco G, Padeletti M, Nuti R, Metra M, et al. Chronic kidney disease and worsening renal function in acute heart failure: different phenotypes with similar prognostic impact? Eur Heart J Acute Cardiovasc Care. 2016 Dec;5(8):534-48.

6 Ostermann M, Zarbock A, Goldstein S, Kashani K, Macedo E, Murugan R, et al. Recommendations on acute kidney injury biomarkers from the acute disease quality initiative consensus conference: a consensus statement. JAMA Netw Open. 2020 Oct 1;3(10): e2019209.

7 Lees JS, Welsh CE, Celis-Morales CA, Mackay D, Lewsey J, Gray SR, et al. Glomerular filtration rate by differing measures, albuminuria and prediction of cardiovascular disease, mortality and end-stage kidney disease. Nat Med. 2019 Nov;25(11):1753-60.

8 van Veldhuisen DJ, Ruilope LM, Maisel AS, Damman K. Biomarkers of renal injury and function: diagnostic, prognostic and therapeutic implications in heart failure. Eur Heart J. 2016 Sep 1;37(33):2577-85.

9 Levey AS, Eckardt KU, Dorman NM, Christiansen SL, Hoorn EJ, Ingelfinger JR, et al. Nomenclature for kidney function and disease: report of a Kidney Disease: Improving Global Outcomes (KDIGO) Consensus Conference. Kidney Int. 2020 Jun;97(6):1117-29.
10 Ricci Z, Cruz DN, Ronco C. Classification and staging of acute kidney injury: beyond the RIFLE and AKIN criteria. Nat Rev Nephrol. 2011 Apr;7(4):201-8.

11 Halloran PF, Famulski KS, Chang J. A probabilistic approach to histologic diagnosis of antibody-mediated rejection in kidney transplant biopsies. Am J Transplant. 2017 Jan; 17(1):129-39.

12 Ronco C, McCullough P, Anker SD, Anand I, Aspromonte N, Bagshaw SM, et al. Cardiorenal syndromes: report from the consensus conference of the acute dialysis quality initiative. Eur Heart J. 2010 Mar;31(6):703-11.

13 Rangaswami J, Bhalla V, Blair JEA, Chang TI, Costa S, Lentine KL, et al. Cardiorenal syndrome: classification, pathophysiology, diagnosis, and treatment strategies: a scientific statement from the american heart association. Circulation. 2019 Apr 16;139(16):e84078.

14 Beldhuis IE, Streng KW, van der Meer P, Ter Maaten JM, O'Connor CM, Metra M, et al. Trajectories of changes in renal function in patients with acute heart failure. J Card Fail. 2019 Nov;25(11):866-74.

15 Damman K, Tang WH, Testani JM, McMurray JJ. Terminology and definition of changes renal function in heart failure. Eur Heart J. 2014 Dec 21;35(48):3413-6. 